\title{
EDITORIAL
}

\section{PROYECCION DE LA INVESTIGACIÓN CUALITATIVA EN ENFERMERÍA EN CHILE}

\author{
PROJECTION OF QUALITATIVE NURSING RESEARCH IN CHILE
}

\author{
Dra. Olivia Sanhueza Alvarado \\ Integrante Comité Editorial Revista Ciencia y Enfermería
}

\begin{abstract}
$\mathrm{R}$ econocer las dinámicas y los esfuerzos de las enfermeras investigadoras en el abordaje cualitativo al interior del país representa un desafío interesante que las propias interesadas debieran realizar.
\end{abstract}

Esto porque la investigación en general en Enfermería se ha ido dando particularmente en el ámbito cuantitativo, con acierto creciente en la obtención de fondos concursables, siendo la investigación cualitativa un método menos exitoso en este sentido, abordaje que a la vez permitiría acrecentar el conocimiento propio de la disciplina.

El origen y desarrollo de la investigación cualitativa en Chile ha estado determinado por la contribución de los programas de postgrado con la formación de magísteres y doctores en Enfermería. Ha quedado pendiente y esto es tal vez una limitación en todo tipo de investigación en Enfermería, la aplicación de sus resultados a la práctica, ya sea hospitalaria o comunitaria, junto con la consolidación de grupos de investigación para hacer investigación cualitativa en el ámbito propio de la disciplina que permita la afirmación de la Enfermería como una disciplina social. Y en este sentido, la evaluación de sus propuestas quedaría inserta en el ámbito de las ciencias humanas y sociales.

En Brasil, la investigación cualitativa en Enfermería surge principalmente de las perspectivas teóricas críticas en ciencias sociales, y de las experiencias y estudios de investigación-acción e investigación-acción participativa. En este país, los estudios cualitativos realizados por las enfermeras a partir de la década de 1989 permitieron que Enfermería fuera vista como una practica social, en la que el paciente, la enfermera y el equipo de Enfermería fueron percibidos como sujetos sociales cuyo producto final consistía en atender las necesidades sociales y en el caso de la salud, la recuperación del individuo o el control de la salud a nivel de la comunidad (1). En esta década comienza también la profundización en materias como: las rela- 
ciones de poder en las instituciones, el proceso de trabajo, las clases sociales y de género, la historia de la salud. Desde ese momento la Enfermería pasa a ser entendida y discutida en cuanto práctica social, y el materialismo histórico pasó a ser la corriente teórica de preferencia (2).

En Chile ha contribuido al uso de enfoques cualitativos, la crítica al paradigma positivista y al modelo biomédico; la influencia de las ciencias sociales en la práctica de la Enfermería y los desarrollos conceptuales alrededor del cuidado de enfermería, influenciado por corrientes como la fenomenología, el interaccionismo y la etnografía y por autores críticos como Foucault, Paulo Freire y humanistas como Humberto Maturana.

Está pendiente aún involucrar a las/os investigadores cualitativos con la práctica, lo que permitiría a los profesionales preocupados con la perspectiva del cuidado que se entrega hacerse cargo de la subjetividad de esta relación y, por ende, de la intersubjetividad en la producción del conocimiento. Las autoras Trentini y Paim (3) proponen la investigación Convergente Asistencial como una nueva modalidad de investigación cualitativa, que tiene como foco la intervención en los escenarios del cuidado de Enfermería, uniendo la practica asistencial con la investigación, lo que permitiría aproximarse a la comprensión de la dimensión existencial de los profesionales y de las personas cuidadas.

\section{REFERENCIAS}

1. Padilha MICS, Kletemberg DF, Gregório VRP, Medeiros Borges L, Borenstein MS. A produção da pesquisa histórica vinculada aos programas de pós-graduação no Brasil, 1972 a 2004. Texto Contexto Enferm. 2007; 16(4): 671-679.

2. Gelbcke FL, Peña, YF, Gallo E. Dialéctica y materialismo histórico: su aproximación al estudio del fenómeno salud/enfermedad. En: Prado MLdo, ed. Investigación cualitativa en enfermería: contexto y bases conceptuales. Washington: Organización Panamericana de La Salud; 2008. p. 147-175.

3. Trentini M, Paim L. Pesquisa convergente assistencial. $2^{\text {a }}$ ed. Florianópolis: Insular; 2004. 